\title{
Universal (Global) Evolutionism
}

\author{
Arkady Ursul \\ Doctor of Philosophical Sciences, Professor Lomonosov Moscow State University \\ (Moscow, Russia) \\ E-mail: ursul-ad@mail.ru \\ ORCID: 0000-0003-2643-5558
}

\begin{abstract}
Tatiana Ursul
Doctor of Philosophical Sciences, Professor National Research Technological

University "MISiS” (Moscow, Russia)

E-mail: ursult@mail.ru

ORCID: 0000-0002-4520-9487
\end{abstract}

\begin{abstract}
In this article investigate a general scientific concept of a global (universal) evolution, in which selforganization of the material systems acts as a common ground and a permanent process of progressive development in the visible Universe. The main problem of research of this type of evolution is seen as a superhighway trajectory of evolutionary processes in the Universe, in which there is a continuous selforganization of the material systems, ranging from the Big Bang and to the social level of evolution, which may have an indefinite continuation of society and nature.

Keywords: evolution, evolutionics, global, global evolutionism, Multiverse, self-organizing, superhighway, universal, universal evolution, Universe
\end{abstract}

Received 1 May 2017; accepted 21 June 2017

Philosophy and Cosmology, Volume 20, 2018: 33-41

DOI: $10.29202 /$ phil-cosm/20/3

\section{Introduction}

Evolutionism in the modern sense is a concept that considers processes not only in their dynamics, motion, change, but also in the development, evolution (this is a broad interpretation of evolutionism, which is not restricted only to the understanding of the development as just gradual quantitative changes). The development refers to the processes, in which there are directed (usually irreversible) changes in the content of certain material entities. The directivity or vector focus of the development manifests itself in the fact that this form of processes can be progressive, regressive, neutral, cyclic development, cycle, etc.

(C) Ursul, Arkady, 2018

(C) Ursul, Tatiana, 2018 
The relationship between the concepts of "evolution" and "development" is not something already established and generally accepted, which draws attention to an interesting and profound monograph by Professor Oleg Bazaluk [Bazaluk, 2014]. He believes that evolution is a complication, and in this we support it, if this concept is attributed to that direction of the vision of evolutionary processes that we attribute to global evolution and which we are considering in this article. Therefore, in fact, although from different authorial positions, we with Oleg Bazaluk consider the same development process, characterized by complication, and basically, if we talk about the Universe as a whole, self-organization as the main evolutionary process.

However, in the general case, the concepts of development and evolution, according to Akop Nazaretyan [Nazaretyan, 2013], are practically synonymous. In this broad vision, development has the same directions that we mentioned above, which already apply not only to global evolution, but also to a common vision of development, the theory of research is sometimes called evolution [Urmantsev, 2009]. Thus, evolution and global evolutionism differ in that the former explores all possible development processes, and universal evolutionism is only a process of complication and self-organization. By the way, universal evolutionism differs from universal history, since this latter focuses only on events and processes that continue to the present and uses primarily a historical approach, rather than an evolutionary one.

\section{Evolutionary and historical approaches}

What is the difference from evolutionary approach from historical? Actually historical approach appears in any science earlier than evolutionary, representing a description of facts, events, mainly of the society as consistently changing in time, i.e. social dynamics in a temporal perspective. As suggested by Leonid Grinin: "In contrast to the historical method evolutionary method of analysis of processes and phenomena considers not all temporary changes, but only the most important qualitative changes and transformations (reorganizations) and evaluates directions of these changes, such as whether they are complication or simplification. Whether it is a new level of evolution or the phenomenon, which is similar to biological adaptive radiation; is historicogenetic connection identified here or it will be impossible to identify it" [Grinin, 2011]. The evolutionary approach also differs from the logical, which was also opposed to the historical method.

Historical approach as an earlier than evolutionary, appears more like a description and in a varying degree like awareness of existential procedural dynamics of human life and at first it was not applied to nature. Evolutionary representations in a varying degree were "woven" into the historical approach, but attention is not focused on them. They have to be identified for the creation of a picture of development of studied process. However, the situation changes now, if science won't be applied to nature, it won't be the history of society but at least socio-natural history. However, historical phenomena, which are in the focus of historical science, are first of all events, facts and processes of the past.

The term "history" is ambivalent: in his ontological understanding history is presented as a chronological sequence of events, as already passed processes of global existence of human society. In epistemological terms history is the science of the phenomenon of man and mankind, of all types and forms of human activity, which are developed in time and space of the historical process. History is represented as the science of the past: historical phenomena, which are in the focus of the study of history, are first of all events, facts and processes of the past. However the past itself is not given to the historian. He obtains information in present, which is left by the past, which he analyses, while the accumulation of information in real forms is the content of progress in history. 
History as a science is presented as a collective memory of mankind's past, it performs the function of preserving the knowledge of civilization and culture. Historians by themselves also believe that retrospective exploration of historical process enables understanding the current state of mankind. It is vital in order to identify prospects and its place in the universe, its fate and the ways and means of survival and further development. However, the history as a science is not focused on the evolution of the studied processes, although does not ignore them, especially in those cases when it is possible to fix the results of the development and its laws at least in the form of trends.

Another feature of the historical approach is associated with the fact that being forced to rely on the facts; the history cannot explore the future, in which these facts do not exist yet. Therefore, the historical approach is mainly based on study of already passed time and only partially of the present, which becomes the past at the moment of completion of the study. Therefore, the history is associated with the science of the past, in which some developmental processes can be identified. However, the temporal focus and the main focus on the past in historical approach prevail over study of evolutionary processes and their future.

We distinguish between the evolutionism as the most extensive ideological and methodological concept and the universal (or global) evolutionism as its part, which is more important in scientific terms. There are conflicts, crises, destruction of systems, etc. in the evolution as the development in the broadest sense (which is studied by evolutionics) as a study area of all evolutionary processes, rather than the global evolutionism, though they have a very wide contact area).

The concepts of development and evolution are virtually equivalent in a broad interpretation, and the evolution includes both quantitative and qualitative, spasmodic changes, and the relationship of progressive and regressive, and other naturally occurring species and forms of development. Such an understanding of the evolution in a broad sense takes place in a number of modern scientific disciplines, such as in astronomy, where the evolution means any directed change in time regardless of whether it is progressive, regressive or any other change. Therefore, in this sense the evolution is identical with the concept of development. It is in this broad interpretation that we consider the notion of evolution, although the latter is considered as the opposite of the concept of revolutionary changes in the development process.

Such an interpretation of evolution is typical of textbooks, which tend to use the "established" meanings of terms. The evolution in this case refers to the process of long, gradual, rather slow changes that lead to the formation of new forms, structures, systems. Such an understanding of evolution in some cases is quite legitimate, but one needs to bear in mind that this interpretation of evolution is in its narrow sense, and it is not practically used in the global evolutionism.

Although the category of revolution is used primarily to characterize fundamental qualitative changes in the social stage of the matter development, but, in the scientific literature, the concept of revolution is used in relation to the transition from one structural level of matter (or stage of evolution) to another, a higher one. Thus, the transition from the inanimate matter to the living one is certainly in this sense an example of revolutionary change, as well as the transition from the living matter to the social one. Therefore, the category of revolution is also applicable to basic qualitative changes in the development of matter.

This broad interpretation of the revolutionary changes often mainly characterizes the ongoing highway of progress in the universe, describing fundamental twists - the emergence of qualitatively new steps and structural levels of matter. Such a split (in the broad and narrow understanding) of the meaning of the category of evolution, in fact, takes place 
in the educational and scientific literature, and we not only state this fact here, but also specifically draw attention to it in order to continue to use mainly the broad understanding of evolution. However, our understanding of the global evolution is related only to the processes that accumulate prior complexity and continue to increase it in the "corridor" of security (preservation) in the process of the self-organization of material systems. The universal (global) evolution is a continuous and end-to-end global (comprehensive) process of progressive development, or complication (self-organization) in our Universe [Bazaluk, 2013, 2014]. In this sense, the superhighway of the global evolution can be considered a special, objectively selected trajectory of permanent self-organization in the Universe.

\section{Worlds of the Universe and global (universal) evolution}

The term "universal" (from the Latin - universalis) means comprehensive, general, and ubiquitous. In this sense, the terms "universal" and "global" (coming from his French meaning) in its content-qualitative, rather than space-geographical sense, are the same. Moreover, such a coincidence, perhaps, makes the choice difficult, which is more dependent on subjective preferences. Yet, we tend to use the concept of global evolution. This is due to the fact that, the idea of evolution and global evolution in the scientific picture of the universe because of the discovery of dark fragments of the universe is essentially being transformed and the entirely new vision of the world is being formed.

That type of evolution, which was often called universal evolution, appears to be not as universal (in the sense of general), as it has recently been considered, because it applies only to a few percent of the universe. In this cognitive situation, it seems appropriate to give preference to the term "global", although we noted above the linguistic equivalence of their use. The global nature is viewed in its "real incarnation", i.e., it is understood that the considered type of evolution covers only a relatively minor - visible fragment of the universe, and it is quite clear that this type of evolution is not specific to the "dark foundation" of the pyramid of self-preservation of matter in the universe (i.e. for the space vacuum and the dark mass), and all the more for the set of mini-universes in the Multiverse [Ursul, 2013].

It is important to distinguish universal evolutionism from planetary evolutionism, whose understanding is usually limited to the scale of the planet Earth and its evolution, and does not focus on singling out permanent self-sustainable trends of self-organization, principles and laws as universals of progressive development of all existing heterogeneous structures of matter.

Several authors include the term "global" to the broad scope of the Universe, or even the Multiverse ${ }^{1}$, i.e. in cosmological cosmic dimension, and there is no common opinion on the use of the discussed concepts. Therefore, the term "global evolutionism" and "universal evolutionism" are being used to the same extent for the description of the permanent progressive evolution in the universe. The concept of "global" itself in this broad sense will not only apply only to the studies of global processes, as is customary in globalistics, studying globalization, global issues and other general planetary processes on our globe. Here, the term "global" can transcend the "planetary" meaning and rush into outer space, as it nearly always occurs in astronomy and space and cosmization areas of research. Therefore, the global research includes global evolutionism, which gives this kind of interdisciplinary research a very broad interpretation.

${ }^{1}$ Multiverse is understood as a totality of simultaneously coexisting mini universes ("local" universes, where our Universe also belongs) together sometimes with a certain pra-ambient from which they originated. 
Preferring the term "global" to "universal" in view of the above considerations, we believe it is appropriate again to draw attention to their linguistic and epistemological equivalence. No one seriously argued one or another preference, and they can be used in the same sense. This "ambiguity" refers not only to the processes of evolution, but also generally to any processes.

There are other terminological options. For example, one can do without the term "global" (but not in case of the issue discussed here). Thus, the evolutionary processes of the planet can be called planetary evolution and of the universe - universal evolution. However, this will not be easy to be done, because the term "global" is already widely used in the scientific literature with respect to universal evolutionary processes. The replacement of the term "global" by "planetary" would lead to a series of forcible substitutions, let us say, globalization for planetarisation, etc., which is obviously unacceptable due to the already well-established scientific tradition and "conceptual inertia".

Moreover, we are not inclined to believe that it is necessary to choose only one meaning of the term "global" and fix it rigidly in globalistics. In principle, it will be impossible to be done, because every researcher has the right to choose. Therefore, these two meanings and the associated criteria will be used in global studies, and it was important to identify the content characteristics of the term "global", which were almost overlooked earlier.

However, when we move from seeing evolutionary processes in the universe to our planet, here the spatial planetary criterion comes into force (the term "geographical" is inappropriate here). In this case, the global evolution coincides with the planetary evolution, where global natural processes first take place, which even though occur on Earth, which completed its astronomical stage of formation about 4.6 billion years ago, but are strongly influenced by the factors of space, the evolution of the Universe. We will proceed from the spatial aspect (criterion) of universal (global) evolution to the content aspect and understanding of what constitutes the phenomenon of global evolution.

The immutability of cosmic vacuum (dark energy) does not give grounds to include it in the process of global permanent self-organization of matter, though this form of matter is related to the process of evolution in the universe because it affects her through antigravity. In our view, the evolution in general and the global evolution in particular take place only in the material part of the universe as a special mini-universe of the Multiverse (where the anthropic cosmological principle is implemented and there is information in inanimate nature).

As far as the dark mass is concerned, it is considered to be a slightly changing fragment of the universe and it is not yet evolution (but its premise, which gives reason to call this process "proto-evolution"). At the same time, gravitating dark mass itself is closer to the evolving material universe, because it consists of some of its constituent particles (at the micro level, the particles may be a thousand times heavier than the proton, whose nature has not been determined yet). It is possible that the dark mass is a certain "transition state" of matter between the space vacuum and baryonic matter as the main forms of matter self-preservation (although there is a hypothesis that it is a form of baryonic matter).

In our view, the evolutionary globalistics will be formed primarily as an interdisciplinary, conceptual approach to the study of global processes and systems in the evolutionary aspect, mainly on the basis of the results obtained in the global (universal) evolutionism. The idea of the development of globalistics as an interdisciplinary field penetrates in various ways, but global evolutionism, which is used in a growing number of scientific disciplines and areas of search, including global studies, can be considered to the most important one. The global evolutionism is a general scientific form of knowledge about the global (universal) 
evolution in which the self-organization of material systems acts as the main permanent process of progressive development in the visible Universe [Moiseev, 1991; Ilyin et al., 2012]. The global evolution is a continuous self-organization of material systems beginning in inanimate nature, going on in living matter and society, and then in the socio-natural form and increasingly covering the material systems of the visible universe. It is understandable that the evolutionary approach in global studies penetrates in other ways too; however, it is not as effective as the application of general scientific principles and approaches, already established in the global evolutionism.

The modern general scientific picture of the world, seeming substantially more systematic than in the last century, has also become more evolutionary, at least for the material fragment of the Universe (although it contains a fundamentally non-evolutionary part in the form of ideas about dark fragments of the universe, and primarily the dark energy, constituting the three-fourths of the material content of the Universe [Astronomy, 2007]².

\section{Superhighway global evolution}

The universal (global) evolution, being characteristic of our visible Universe, highlights only one thing from all the directions of development - its main continuous progressive direction as the trajectory of permanent self-organization, as it only leads to higher and higher levels and stages of development of material systems. It is on this trajectory, called the superhighway, that those forms and levels of development are found, which are not interrupted, and extend continuously, exist during the whole time of the universe evolution. It is for them that the principles, trends and patterns of progressive development are identified, which are of universal and invariant nature, thanks to them, we can understand and explain the origin of life and intelligent beings. These laws also enable us to predict the further interaction of the social stage of evolution with the nature of the earth and the cosmos. The development of global evolutionism, as an interdisciplinary scientific concept and a major fragment of the scientific picture of the world, is in line with the studies that are the core of the generation and synthesis of scientific knowledge, the formation of a unified science of the future.

The superhighway is typical of a "preserving" (and for higher stages of evolution - safe) type of progressive permanent development when, for example, the individual, selected for one reason or another, biological or social systems remain intact during the revolution and continue to exist through self-organization after another bifurcation. The permanent progressive evolution is a "safe" type of self-organization, leading to the emergence of new, higher structural levels and stages of the development of material systems.

However, there is a kind of selection on the highway of this revolution (superhighway) when the material structures, enriching their information content, only gain "the ability of the future". After all interactions (including crisis conflict ones), these structures go to co-evolutionary interactions with the other material structures, surrounding them, which constitute the environment of existence and development. This is "required" by the strange attractor of global evolution, influencing positively on the process of self-organization of the evolving structure: as non-co-evolutionary structures collapse after the phase of bifurcation. The co-evolutionary and thereby preserved material structures, in a very narrow (and more

${ }^{2}$ Dark matter has two forms - a dark mass, or invisible gravitating substance constituting $22 \%$ of the energy density of the universe and dark energy. The contribution of this form of matter in the density of universe energy makes about $74 \%$, while ordinary matter accounts for only $4 \%$, including the stars $-1 \%$. 
increasingly shrinking) range or "security corridor", have the opportunity to continue the evolutionary "relay race" on the superhighway of the global evolution.

Are there crises and disasters on the superhighway itself - the question is not simple, and for the present it has no clear answer. There are various types and forms of development around the superhighway, and this is quite understandable. Moreover, the superhighway itself exists at the expense of the rest of the universe - it results from the fundamental provisions of synergy and cosmology. Yet it can be assumed that only the permanent accumulation of the information content of self-organizing systems happens on the superhighway, purely logically, there should not be "evolutionary informational failures" negative entropy loss, as it is fraught with the disappearance of the superhighway, which could not be interrupted, as it existed from the very the beginning of the Big Bang and exists now. Most likely, it should be assumed that the superhighway provides progressive development without crises and catastrophes, providing over billions of years the preservation and increase of negative entropy and information content of evolving systems. At the same time, the superhighway itself is the main attractor of this sustainable and crisis-free type of progressive development, which is implemented through self-organization. However, this is just the statement of a question, and we understand that there need to be further research and discussion.

The most common vision of global evolutionism in recent years is associated with synergy, which transferred many of its principles and laws from inanimate nature to the worlds of the living and the social. The synergetic approach in the study of global evolutionism uses the concepts and methods of the mentioned scientific field to identify the laws of selforganization of systems as spontaneous appearance of complex ordered structures. There are other approaches to the study of universal evolution.

The global evolution is still poorly explored and the most interesting issue of all science in general and therefore it increasingly attracts the attention of scientists. It becomes clear that the foundation of the universe itself and its evolution have some principles and trends, with which that part of the universe, which gained intelligence and socio-cultural form of its existence and evolution, should comply as well.

The planetary evolutionary processes, this way or another, are related to the main trajectory of the continuous self-organization of material systems in the universe, which we called the superhighway of global or universal evolution. Moreover, it is important to figure out how they relate to it, and, above all, whether they fit into this superhighway. It is known that, in the course of the global evolution, the process of co-evolution (i.e., co-development, conjugate development without destroying the interacting systems) of two or more systems in their interaction that leads to both the harmonization of the relations between these systems and to the general co-evolutionary synergetic effect. Therefore, on the planetary level of organization, one can talk about co-evolution of the planet shells - geospheres, without which there would not have been the structural and functional unity of the planet as the existing system [Ilyin \& Ivanov, 2009].

As the human activity has become a planetary geological factor and, thus, from the point of view of globalistics - global activity, it has gone beyond that earthly shell, in which it emerged and evolved. It is meant here that it has gone beyond all geospheres, including the limits of the biosphere, both in space and deeper into the planet. So as to preserve the natural environment, surrounding mankind, in a broad sense (i.e. not only the biosphere), it is important to carry out global activities within the carrying capacity of ecosystems the concept that previously made little sense in terms of the biosphere. This concept is already expanding its content, and it will have to be supplemented by such concepts as 
"geological carrying capacity" and "carrying capacity of cosmo-ecosystems." It is even more dangerous to go beyond these "carrying capacities" than in the case of the violation of carrying capacities of ecosystems in the biosphere. This is explained by the fact that there is a powerful regulator and the stabilizer of the environment in the biosphere- biota, and outside the Earth's biosphere, especially in space, the natural homeostatic factors and mechanisms like Le Chatelier's principle are not as efficient and cannot therefore be taken into account as the methods of systems self-preservation.

If one goes beyond the temporal limits of what is going on in the last decades or even centuries into the broader time periods, one can conceive quite differently global processes and emerging global systems (structures). The evolutionary vision reveals certain directed transformations of all global processes, structures created with their help (which have not only global spatial scale, but also global importance) and even the emergence of new ones, mainly as a result of human activity and the development of socio-natural stage of evolution. From the standpoint of the evolutionary approach used here all global processes and systems, as it has been noted, may have progressive, regressive or other orientation of development.

\section{Conclusion}

This is our vision of global (universal) evolution. This grand planetary-cosmic process was developing with people participation and has the potential to extend human activity into the Universe.

Global (universal) evolutionism is a crossroad of ideas, concepts, challenges and hypotheses. It focuses on one of the most topical issues - evolutionary processes in the Universe, that is, on permanent self-organization of material systems which lies at the basis of sustainable progressive development of organic and non-organic nature and, later, of social evolution. Further development is seen not merely as self-development of the social matter but mainly as co-evolution of the social sphere and natural environment in the Universe.

Global evolutionism is now included in such an important interdisciplinary trend of all modern science as global studies in their broad understanding. Global studies has already started to form as a special global cluster of scientific knowledge, which includes globalistics, global evolutionism, separate global disciplines (such as the global economy, global ecology, etc.) [Ilyin et al., 2016].

However, being included in the global studies, global evolutionism claims to be a general scientific conceptual and methodological basis for all modern and, especially, future science. The concept of universal (global) evolution is one of the "epistemological attractors" in increments of scientific knowledge, which not only receives information from other sciences, but also is a kind of conceptual core, the part of the scientific picture of the world of the $21^{\text {st }}$ century, synthesizing knowledge in the above-mentioned areas of scientific research.

\section{메] References}

Astronomy: Century 21 ${ }^{\text {st }}$. Ed.-compiler Vladimir Surdin, Fryazino, 2007: 29, 426 (In Russian). Bazaluk, Oleg. Theory of Evolution. Philosophy and Cosmology. Vol. 15, 2015: 25-33.

Bazaluk, Oleg. The Basic Postulates of the Universal Evolution Model "Evolving matter". Philosophy and Cosmology. Vol. 14. 2013: 11-20.

Bazaluk, Oleg. The Theory of Evolution: From a Space Vacuum to Neural Ensembles and Moving Forward. Kiev: MFKO, 2014: 22-72 (In Russian).

Grinin, Leonid. Evolution: cosmic, biological, social opportunities of single paradigm. 
Globalistics as a field of research and the world of teaching. Vol. 5. Eds. Igor Abylgaziev, and Ilya Ilyin. Moscow: Moscow State University, 2011:106 (In Russian).

Ilyin, Ilya, Arkady Ursul, and Tatiana Ursul. Global evolutionism: Ideas, issues, hypotheses. Moscow: Moscow State University, 2012. (In Russian).

Ilyin, Ilya, Arkady Ursul, and Tatiana Ursul. Global Studies. Saarbrücken: Lambert Academic Pablishing, 2016.

Ilyin, Ilya, and Aleksey Ivanov. Introduction to Global Ecology. Moscow: Moscow State University, 2009: 94-96 (In Russian).

Moiseev, Nikita. Universal evolutionism (Position and effect). Questions of Philosophy. 3, 1991: 12-28 (In Russian).

Nazaretyan, Akop. Nonlinear future. Metahistoric, synergetic and cultural-psychological prerequisites for global prediction. Moscow: Publisher MBA, 2013. (In Russian).

Urmantsev, Ynir. Evolutionics as a general development theory of natural systems, society, thinking. Moscow, 2009. (In Russian).

Ursul, Arkady. Dark matter and the global evolution. Philosophical Thought, 6, 2013: 96-170 (In Russian). 